VOL. $19(1978), 197-203$.

\title{
Residual finiteness of cyclic extensions of certain free products
}

\section{Benjamin Baumslag, Narain Gupta, and Frank Levin}

\begin{abstract}
We prove that a cyclic extension of a free product of finite p-groups is residually finite, $p$ a fixed prime. The question of cyclic extensions of free products of arbitrary cyclic groups remains open.
\end{abstract}

\section{Introduction}

In [2] Gilbert Baumslag proved that any finitely generated cyclic extension of a free group is residually finite. This raises the question of cyclic extensions of arbitrary free products of cyclic groups. In this note we prove that if $H$ is a free product of finite $p$-groups for the same prime $p$, then any cyclic extension of $H$ is residually finite. This follows from a more general result: if $H$ is a free product of finitely generated nilpotent groups, none of which are infinite cyclic, and all of whose torsion subgroups, if any, are $p$-groups for the same prime $p$, then any cyclic extension of $H$ is residually finite (Theorem A). The proof is similar to that used by Gilbert Baumslag for cyclic extensions of free groups. An important difference is that we do not require that the extension be finitely generated.

Our proof requires that $H$ be residually nilpotent, which is not, for example, the case when the primes involved are different. In this direction however, we have a partial result (a special case of Theorem $B$ ): Let $H$ be a free product of finitely generated $p$-groups where, with the

Received 31 July 1978. The second author was supported in part by a grant from the National Research Council of Canada. The authors thank Dr P.M. Neumann for a useful discussion during the revision of an earlier draft of this paper. 
possible exception of one of the primes involved, there are at most finitely many factors to each prime. Then cyclic extensions of $H$ are residually finite. The problem of arbitrary free products remains open.

\section{Proofs}

For each positive integer $c$ let $\Pi\left(c, H_{i}, I\right)$ denote the cth nilpotent product of the groups $H_{i}, i \in I$ (see, for instance, [3]). In addition we will allow $c$ to take on the value $\infty$ by defining TT $\left(\infty, H_{i}, I\right)$ to be the free product of the groups $H_{i}, i \in I$.

DEFINITION. Let $G$ be a cyclic extension of $H, G=\langle t\rangle$, where $H=\prod\left(c, H_{i}, I\right)$, as above, such that for each $i \in I$ there exist elements $j \in I$ and $h \in H$ satisfying $H_{i}^{t}=H_{j}^{h}$. Then $G$ is called a cof extension of $H$ relative to the decomposition $\prod\left(c, H_{i}, I\right)$. In the special case where $H_{i}^{t}=H_{j}$ for each $i \in I$ we call $G$ a reguzar cof extension relative to the given decomposition.

Given a cof extension we define a t-action on $I$ by defining $i t=j$ if $H_{i}^{t}$ is conjugate in $H$ to $H_{j}$. We denote the orbit containing $i$ by $[i]$; that is,

$$
[i]=\left\{i t^{r} \mid r \in \mathbb{Z}\right\} \text {. }
$$

More generally, if $S \subseteq I$, we define $[S]=\bigcup_{i \in S}[i]$.

We recall that the orbits form a partition of $I$.

The following result is almost immediate.

LEMMA 1. Let $G$ be a cof extension of $H$ relative to TT $\left(c, H_{i}, I\right)$ and let $S$ be any subset of $I$. Define $K$ to be the normal closure in $H$ of the subgroup $\Pi\left(c, H_{i}, I \backslash[S]\right)$. Then $K$ is normal in $G$ and $G / K$ is a cof extension of $H / K \cong \prod T\left(c, H_{i},[S]\right)$.

COROLLARY 1. To show that $G$ is residually finite it may be assumed, with no loss in generality, that the number of orbits is finite. 
Proof. Let $g \neq l$ be an element of $G$. Then $g=t^{r} h$ for some integer $r$ and $h \in H$, and $h$ is expressible as the product of elements from say $H_{i_{1}}, H_{i_{2}}, \ldots, H_{i_{k}}$. Let $s=\left\{i_{1}, i_{2}, \ldots, i_{k}\right\}$ and construct $K$ as in the lemma. Then $g \mid K$, so if $G / K$ is residually finite there is a finite homomorphic image of $G / K$, and hence of $G$, in which $g$ is nontrivial. Since the number of orbits of $H / K$ in $G / K$ is finite, the result follows.

COROLLARY 2. Let $G$ be a cof extension of $H$ as above, and suppose that the components $H_{i}$ of $H$ are finitely generated and residually finite. Furthermore, suppose that all orbits are finite. Then $G$ is residually finite.

Proof. By Corollary 1, we may assume that the number of orbits is finite, and hence, $H$ is finitely generated. Since a free product of residually finite groups is residually finite. (see [1] for a short proof), $G$ is a cyclic extension of a finitely generated residually finite group and, hence, is residually finite.

An interesting application of Corollary 2 is to the case where $H$ is the free product of finite groups $H_{i}$ such that for each integer $n$ at most a finite number of the groups $H_{i}$ are of order $n$.

LEMMA 2. Let $G$ be a regular cof extension of $H$. To show that $G$ is residually finite it may be assumed, with no loss in generality, that every orbit is finite.

Proof. We consider the case where $I$ contains only one infinite orbit, since the more general case requires only further notation but no change in proof. Thus, let

$$
H=\prod\left(c, H_{i}, \mathbb{Z}\right)
$$

with $\left(B_{0}\right)^{t^{r}}=H_{r}$, for all integers $r$.

For any integer $m$ greater than 1 let

$$
H(m)=\prod\left(c, H_{i},\{1,2, \ldots, m\}\right)
$$

and define the action of an element $\bar{t}$ on $H(m)$ by 


$$
H_{i}^{\bar{t}}=H(\widehat{i+1})
$$

where $\widehat{i+1}$ is the least positive residue congruent to $i+1$ modulo $m$. In this way we obtain a cof extension $G_{m}$, say, of $H(m)$.

Now $t^{i-\hat{i}}$ induces an isomorphism $\psi_{i}$ of $H_{i}$ onto $H_{i}$. The maps $t \rightarrow \bar{t}$ and $\psi_{i}$ induce a homomorphism $\theta_{m}$, say, of $G$ onto $G_{m}$. For each $g$ in $G, g \neq I$, we can choose $m$ sufficiently large so that $g^{\theta}{ }_{m} \neq 1$, and this establishes the result.

Let $\gamma_{\mathcal{C}}(H)$ denote the cth term of the lower central series of $H$. We then have the following result.

LEMMA 3. Let $G$ be a cof extension of $H=\prod\left(\infty, H_{i}, I\right)$. Then for any integer $c, G / \gamma_{c}(H)$ is a regular cof extension of $\bar{H}=H / \gamma_{c}(H)$ with respect to the decomposition into a cth nilpotent product with factors conjugate to the $H_{i} \gamma_{c}(H) / \gamma_{c}(H)$.

Proof. We denote the images under the natural map $G \rightarrow G / \gamma_{c}(H)$ by bars. Once again we assume that $I$ is a single infinite orbit, the other cases being similar. Thus, suppose (1) holds and that

$$
H_{0}^{t^{r}}=H_{r}^{h}
$$

where $h_{x}$ is an element of $H$. Then

$$
\bar{H}=\prod\left(c, \bar{H}_{i}, \mathbb{Z}\right)
$$

Using generators and defining relators it is easy to see that if a group $A$ is the cth nilpotent product of groups $A_{i}$ of nilpotent class at most $c$, then for arbitrary elements $g_{i}$ of $A, A$ is also the cth nilpotent product of the groups $A_{i}^{g_{i}}$. Thus if we put $\left.\overline{\left(H^{h}\right.}\right)=B_{i}$, then

$$
\bar{H}=\prod\left(c, B_{i}, \mathbb{Z}\right)
$$

and $\bar{G}$ is a regular cof extension of $\bar{H}$ relative to (2). 
THEOREM A. Let $G=H(t)$ be a cyclic extension of $H=\prod\left(\infty, H_{i}, I\right)$ where each $H_{i}$ is a finitely generated nilpotent group, not infinite cyclic, whose torsion subgroup, if any, is a p-group for the same prime $p$. Then $G$ is residually finite.

Proof. It is easy to see that the non-infinite-cyclic maximal nilpotent subgroups of $H$ are the conjugates of the $H_{i}$ in $H$. Thus $G$ is a cof extension of $H$. By Corollary l it suffices to deal with the case of a finite number of orbits only.

Now if $g$ is a non-unit element of $G$ we must find a normal subgroup of finite index which excludes $g$. This is easily done if $g$ is not in $H$, so we may assume that $g \in H$. By a result of Mal'cev [4], $H$ is residually nilpotent. Thus there exists an integer $c$ such that $g \vDash \gamma_{c}(H)$. By Lemma $3, \bar{G}=G / \gamma_{c}(H)$ is a regular cof extension, and by Lemma 2 we may assume that $\bar{G}$ has finite orbits only. Thus, $\bar{H}=H / \gamma_{c}(H)$ is a finitely generated nilpotent group and, hence is residually finite. Since a cyclic extension of a finitely generated residually finite group is residually finite, the result follows.

To prove Theorem B we shall need the following obvious result.

LEMMA 4. Let $G$ be a cof extension of $H=\prod\left(c, H_{i}, I\right)$ and let $i_{1}, i_{2}, \ldots, i_{k}$ be elements of I from distinct finite orbits of cardinality $m_{1}, m_{2}, \ldots, m_{k}$, respectively. Let $m=m_{1} \ldots m_{k}$. Then if $s=t^{m}, H(s)$ is a cof extension of $H$ in which the orbits containing $i_{1}, \ldots, i_{k}$ each consist of precisely one element. Furthermore, since $H\langle s\rangle$ is of finite index in $G, G$ is residually finite if and only if $H\langle s\rangle$ is.

THEOREM B. Let $p$ be a given prime and $H$ a free product of finitely generated groups $H_{i}$, none of which is infinite cyclic. Suppose that if $H_{i}$ is infinite, it is nilpotent with torsion subgroup (if any) a p-group. Further, suppose that for each integer $n$ not a power of $p$ the number of factors $H_{i}$ of order $n$ is finite. Then cony cyclic extension $G$ of $H$ is residually finite. 
Proof. Clearly $G$ is a cof extension. Let $Q$ be the free product of all the infinite $H_{i}$ and of those which are p-groups and $P$ be the free product of the remaining factors. Then $H=P * Q$. To show that $G$ is residually finite it suffices to show that a non-trivial element $g$ in $H$ is non-trivial in some finite image of $G$. We may assume, by Corollary 1 , that $G$ has finitely many orbits and by Lemma 4 , that if $H_{i}$ is a factor of $P$, then the orbit containing $i$ consists precisely of $i$.

If $Q^{H}$ denotes the normal closure of $Q$ in $H$, then $G / Q^{H}$ is residually finite by Lemma 1 and Corollary 2, while $G / P^{H}$ is residually finite by Lemma $I$ and Theorem A. Thus, we may assume that $g$ is in $P^{H} \cap Q^{H}$. We define $Z(g)$ to be the number of occurrences of elements from the factors of $P$ when $g$ is expressed in normal form, that is, $Z(g)$ is the syllable length of $g$ with respect to the factors of $P$. The remainder of the proof is by induction on $Z(g)$ in any group of the type described in the theorem.

If $Z(g)=0$, then $g \notin P^{H} \cap Q^{H}$. Thus, assume the result true for $Z(g)$ less than $n$ and let $Z(g)=n$. Suppose that $g$ involves in its normal form elements from a factor, $H_{I}$ say, of $P$. Let $K$ be the normal closure in $H$ of all the $H_{i}$ with the exception of $H_{1}$. We note, by Lemma 1 , that $K$ is normal in $G$ and $H / K$ is isomorphic to the finite group $H_{1}$. It is easy to see that $K$ is a free product satisfying the same hypotheses that $H$ does in the statement of the theorem. Clearly $g$ is in $K$, and when $g$ is expressed in normal form in $K, Z(g)<n$. Now $\bar{H}=K(t)$ is an extension of the type described in the theorem, so by the induction hypothesis there is a finite factor of $\bar{H}$ excluding $g$. Since $\bar{H}$ is of finite index in $G$, the result follows.

\section{References}

[1] Benjamin Baumslag, Marvin Tretkoff, "Residually finite HNN extensions", Comm. AZgebra 6 (1978), 179-194. 
[2] Gilbert Baumslag, "Finitely generated cyclic extensions of free groups are residually finite", Bull. Austral. Math. Soc. 5 (1971), $87-94$.

[3] Wilhelm Magnus, Abraham Karrass, Donald Solitar, Combinatorial group theory: Presentations of groups in terms of generators and relations (Pure and Appl. Math. 13. Interscience [John Wiley \& Sons], New York, London, Sydney, 1966. Second, revised edition, Dover, New York, 1976).

[4] A.1. Mal'cev, "Generalized nilpotent algebras and their adjoint groups", Amer. Math. Soc. Tronst. (2) 69 (1968), 1-21.

Department of Mathematics,

Imperial College of Science and Technology,

London,

England;

Department of Mathematics, University of Manitoba,

Winnipeg,

Manitoba,

Canada;

Institut für Mathematik, Ruhr-Universität Bochum, Bochum,

Federal Republic of Germany. 